\title{
1974 CONJECTURE OF ANDREWS ON PARTITIONS
}

\author{
PADMAVATHAMMA and M. R. SALESTINA
}

Received 13 June 2002

\begin{abstract}
The case $k=a$ of the 1974 conjecture of Andrews on two partition functions $A_{\lambda, k, a}(n)$ and $B_{\lambda, k, a}(n)$ was proved by the first author and Sudha (1993) and the case $k=a+1$ was established by the authors (2000). In this paper, we prove that the conjecture is false and give a revised conjecture for a particular case when $\lambda$ is even.
\end{abstract}

2000 Mathematics Subject Classification: 11P82, 05A19.

1. Introduction. Andrews [3] proved a general theorem from which the well-known Rogers-Ramanujan identities, Gordon's theorem [7], the Göllnitz-Gordon identities [6] and their generalization [1], Schur's theorem and its generalization [10] could be deduced. In 1969, Andrews [2] proved the following theorem.

THEOREM 1.1 [2, Theorem 2]. If $\lambda, k$, and a are positive integers with $\lambda / 2 \leq a \leq k$, $k \geq 2 \lambda-1$, then for every positive integer,

$$
A_{\lambda, k, a}(n)=B_{\lambda, k, a}(n),
$$

where $A_{\lambda, k, a}(n)$ and $B_{\lambda, k, a}(n)$ are defined as follows.

DEFINITION 1.2. For an even integer $\lambda$, let $A_{\lambda, k, a}(n)$ denote the number of partitions of $n$ into parts such that no part which is not equivalent to $0(\bmod \lambda+1)$ may be repeated and no part is equivalent to $0, \pm(a-\lambda / 2)(\lambda+1) \bmod [(2 k-\lambda+1)(\lambda+1)]$. For an odd integer $\lambda$, let $A_{\lambda, k, a}(n)$ denote the number of partitions of $n$ into parts such that no part which is not equivalent to $0(\bmod ((\lambda+1) / 2))$ may be repeated, no part is equivalent to $\lambda+1(\bmod 2 \lambda+2)$, and no part is equivalent to $0, \pm(2 a-\lambda)((\lambda+$ $1) / 2) \bmod [(2 k-\lambda+1)(\lambda+1)]$.

DEFINITION 1.3. Let $B_{\lambda, k, a}(n)$ denote the number of partitions of $n$ of the form $b_{1}+\cdots+b_{s}$ with $b_{i} \geq b_{i+1}$, no part which is not equivalent to $0(\bmod \lambda+1)$ is repeated, $b_{i}-b_{i+k-1} \geq \lambda+1$ with strict inequality if $\lambda+1 / b_{i}, \sum_{i=j}^{\lambda-j+1} f_{i} \leq a-j$ for $1 \leq j \leq(\lambda+1) / 2$, and $f_{1}+\cdots+f_{\lambda+1} \leq a-1$, where $f_{j}$ is the number of appearances of $j$ in the partition.

Since Schur's theorem [10] is the case $\lambda=k=a=2$, it is not a particular case of Theorem 1.1 as $k \geq 2 \lambda-1$ is not satisfied. Hence Andrews [2] conjectured that Theorem 1.1 may be still true if $k \geq \lambda$. In fact, he gave a proof of this result [4].

In the conclusion of [4], Andrews stated the following two conjectures. 
Conjecture 1.4. For $\lambda / 2<a \leq k<\lambda$, let $n^{c}=(k+\lambda-a+1)(k+\lambda-a) / 2+(k-\lambda+$ 1) $(\lambda+1)$. Then

$$
\begin{aligned}
& B_{\lambda, k, a}(n)=A_{\lambda, k, a}(n) \quad \text { for } 0 \leq n<n^{c}, \\
& B_{\lambda, k, a}(n)=A_{\lambda, k, a}(n)+1 \quad \text { for } n=n^{c}
\end{aligned}
$$

CONJECTURE 1.5. For all positive integers $n, A_{4,3,3}(n)=B_{4,3,3}^{0}(n)$, where $B_{4,3,3}^{0}(n)$ denotes the number of partitions of $n$ enumerated by $B_{4,3,3}(n)$ with the added restrictions:

$$
\begin{gathered}
f_{5 j+2}+f_{5 j+3} \leq 1 \quad \text { for } j \geq 0 \\
f_{5 j+4}+f_{5 j+6} \leq 1 \quad \text { for } j \geq 0 \\
f_{5 j-1}+f_{5 j}+f_{5 j+5}+f_{5 j+6} \leq 3 \quad \text { for } j \geq 1 .
\end{gathered}
$$

Conjecture 1.5 is designed to show that when the condition $k \geq \lambda$ is removed with some additional restrictions on the summands, some partition identities can be obtained in a few cases. In 1994, Andrews et al. [5] proved Conjecture 1.5.

The first author and Sudha [9] have proved the case $k=a$ of Conjecture 1.4 while the authors in [8] have established the case $k=a+1$ of Conjecture 1.4. The objective of the present paper is to prove that Conjecture 1.4 is false if $n$ exceeds $(2 k-a-\lambda / 2+1)(\lambda+$ 1 ) for even $\lambda$ and $k \geq a+2$. For odd $\lambda$, we have verified and checked that Conjecture 1.4 is false when $\lambda=11, k=9$, and $a=6$. We also give the following revised conjecture for a particular case when $\lambda$ is even.

REVISED CONJECTURE 1.6. Let $\lambda$ be even, $a-\lambda / 2=1, \theta=k-a, \theta(\theta-1) / 2<[a-$ $\lambda / 2](\lambda+1)$, and $0 \leq \theta \leq \lambda / 2-3$. Then

$$
\begin{aligned}
& B_{\lambda, k, a}(n)=A_{\lambda, k, a}(n) \quad \text { for } n<\left(2 k-a-\frac{\lambda}{2}+1\right)(\lambda+1), \\
& \quad B_{\lambda, k, a}(n)=A_{\lambda, k, a}(n)+B_{\lambda, k, a}(x), \\
& \text { where } n=\left(2 k-a-\frac{\lambda}{2}+1\right)(\lambda+1)+x, 0 \leq x \leq \frac{\theta(\theta-1)}{2} .
\end{aligned}
$$

These results support (i) Andrews' contention that $k \geq \lambda$ is essential for the truth of Theorem 1.1 and (ii) his belief that Theorem 1.1 was the best possible one, but his conjecture about first counterexamples when $k \geq \lambda$ is false.

2. Preliminaries. Let $P_{B_{\lambda, k, a}}(n)$ and $P_{A_{\lambda, k, a}}(n)$ denote the sets of partitions enumerated by $B_{\lambda, k, a}(n)$ and $A_{\lambda, k, a}(n)$, respectively. Let $P_{A}^{\prime}(n)$ (resp., $P_{B}^{\prime}(n)$ ) denote the set of partitions enumerated by $A_{\lambda, k, a}(n)$ (resp., $\left.B_{\lambda, k, a}(n)\right)$ but not by $B_{\lambda, k, a}(n)\left(\operatorname{resp} ., A_{\lambda, k, a}(n)\right.$ ). $\pi \in P_{A}^{\prime}(n)$ implies that it violates one of the conditions on $f^{\prime} s$ or $b^{\prime} s$. Let $S_{j}(j=$ $1,2, \ldots, \lambda / 2)$ denote the condition $\sum_{i=j}^{\lambda-j+1} f_{i} \leq a-j$, let $S$ denote the condition $\sum_{i=1}^{\lambda+1} f_{i} \leq$ $a-1$, and let $S^{*}$ be the condition on $b^{\prime} s$.

Let $(2 k-a-\lambda / 2+1)(\lambda+1) \leq n<(2 k-a-\lambda / 2+1)(\lambda+1)+\theta(\theta-1) / 2$, where $\theta(\theta-1) / 2<(a-\lambda / 2)(\lambda+1)$ and $\theta=k-a$. Then

$$
P_{B}^{\prime}(n)=Q^{1} \cup \cdots \cup Q^{a-1} \cup R(n),
$$


where for $1 \leq i \leq a-1$,

$$
\begin{aligned}
Q^{i}= & \left\{\pi \in P_{B}^{\prime}(n):\left(a-\frac{\lambda}{2}\right)(\lambda+1) \text { appears } i \text { times }\right\}, \\
R(n)= & \left\{\left(2 k-a-\frac{\lambda}{2}+1\right)(\lambda+1)+\pi: \pi\right. \text { is a partition of } \\
& \left.n-\left(2 k-a-\frac{\lambda}{2}+1\right)(\lambda+1) \text { into parts with } C\right\} .
\end{aligned}
$$

Here $C$ stands for "subjected to the conditions in the definition of B." Clearly, $\# R(n)=$ $B_{\lambda, k, a}[n-(2 k-a-\lambda / 2+1)(\lambda+1)]$.

From the method explained in [8,9], it follows that the partitions violating $S_{1}, \ldots, S_{\lambda / 2}$ will be mapped onto $Q^{1} \cup \cdots \cup Q^{a-1}$. If $a-\lambda / 2=1$, then $S$ reduces to $S_{1}$. As such, any contribution to $R(n)$ can come only from those partitions of $P_{A}^{\prime}$ which violate $S^{*}$ but do not violate any of $S_{1}, \ldots, S_{\lambda / 2}$. For the counterexample in Section 3, we enumerate separately the partitions counted by $R(n)$. If there are no partitions of $n$ violating only $S^{*}$, then for such $n$, we have that $P_{A}^{\prime}(n)$ is the union of the partitions violating $S_{1}, \ldots, S_{\lambda / 2}$ and $Q^{a-1}$ is the set containing $a-1$ times $\lambda+1$. This set is identified with the first stage of $S_{1}$ where all the parts from $1, \ldots, \lambda$ appear. $Q^{a-2}$ will be the union of the two sets, one containing $a-2$ times $\lambda+1$ and the other containing $a-2$ times $\lambda+1$ plus a part between 1 and $\lambda$. These two sets are, respectively, identified with the first stage of $S_{2}$ where all the parts from $2, \ldots, \lambda-1$ appear, and the second stage of $S_{1}$ in which all the parts except one part from $1, \ldots, \lambda$ appear and so on.

3. Counterexample. Let $\lambda=12, k=11, a=7, \theta=4, a-\lambda / 2=1, \theta(\theta-1) / 2=6<$ $(a-\lambda / 2)(\lambda+1)=13$, and $n^{c}=136$. In this case,

$$
\begin{gathered}
S_{\lambda / 2}=S_{6}: f_{7}+f_{6} \leq 1, \quad S_{5}: f_{8}+f_{7}+f_{6}+f_{5} \leq 2, \quad S_{4}: f_{9}+\cdots+f_{4} \leq 3, \\
S_{3}: f_{10}+\cdots+f_{3} \leq 4, \quad S_{2}: f_{11}+\cdots+f_{2} \leq 5, \quad S_{1}: f_{12}+\cdots+f_{1} \leq 6, \\
S: f_{13}+\cdots+f_{1} \leq 6 ; \\
P_{B}^{\prime}(n)=Q^{1} \cup \cdots \cup Q^{6} \cup R(n),
\end{gathered}
$$

where $Q^{i}=\left\{\pi \in P_{B}^{\prime}(n): 13\right.$ appears $i$ times $\}, 1 \leq i \leq 6$, and $R(n)=\{130+\pi: \pi$ is a partition of $n-130$ into parts with $C\}$. Here $\# R(n)=B_{12,11,7}(x)$, where $x=n-130$. We now prove

$$
\begin{gathered}
B_{12,11,7}(n)=A_{12,11,7}(n), \quad n<130, \\
B_{12,11,7}(n)=A_{12,11,7}(n)+B_{12,11,7}(x), \quad n=130+x, 0 \leq x<6, \\
B_{12,11,7}(136)=A_{12,11,7}(136)+B_{12,11,7}(6)-1=A_{12,11,7}(136)+3,
\end{gathered}
$$

since $B(6)=4$ as $6,5+1,4+2$, and $3+2+1$ are the only relevant partitions of 6 enumerated by $B$. 
Proof of (3.2), (3.3), and (3.4). Equation (3.2) follows from [8]. We now prove that for $1 \leq n<136$, there are no partitions of $n$ violating only $S^{*}$ and that

$$
18+17+16+15+14+12+11+10+9+8+6
$$

is the only partition of 136 violating only $S^{*}$.

In $[8,9]$ we have shown that for $n<130$, if a partition violates $S^{*}$, then it violates either $S$ or $S_{1}$. However, for $130 \leq n \leq 136$, we now investigate such partitions.

If a partition violates $S^{*}$, then there exist a partition

$$
n=b_{1}+\cdots+b_{i}+\cdots+b_{i+10}+\cdots+b_{s}
$$

and an integer $i$ with $b_{i}-b_{i+10}<13$. We get the following possibilities.

CASE 1. If $b_{i+10} \geq 13$, then the number being partitioned is greater than or equal to

$$
\begin{aligned}
& \left(12+x_{11}\right)+\cdots+\left(12+x_{1}\right)+\cdots, \\
& 11(12+1), \quad \text { where } x_{11}-x_{1}<13 .
\end{aligned}
$$

If (3.7) contains the part 13 more than 6 times, then it violates $S$. Let $x \leq 6$ be the number of 13's and let $y$ denote the number of terms greater than 13 in (3.7) so that $x+y=11$. Then (3.7) becomes

$$
13 x+(12+2)+\cdots+(12+11-x)=11(13)+\frac{(11-x)(11-x-1)}{2} .
$$

Let $n^{c}$ denote the $n$ in the conjecture. If $k=a+\theta$, then

$$
\begin{aligned}
n^{c} & =\left(2 k-a-\frac{\lambda}{2}+1\right)(\lambda+1)+\frac{\theta(\theta-1)}{2} \\
& =k(\lambda+1)+\left(k-a-\frac{\lambda}{2}+1\right)(\lambda+1)+\frac{(k-a)(k-a-1)}{2} \\
& <k(\lambda+1)+\left(k-a-\frac{\lambda}{2}+1\right)(\lambda+1)+\frac{(k-x)(k-x-1)}{2} \\
& <k(\lambda+1)+\frac{(k-x)(k-x-1)}{2} \text { since } k-a-\frac{\lambda}{2}+1<0 .
\end{aligned}
$$

In this case, we have that $n^{c}<11(13)+(11-x)(11-x-1) / 2$.

CASE 2. Let $b_{i+10}<13$ and $b_{i}<13$. Then (3.6) violates $S_{1}$.

CASE 3. Let $b_{i+10}<13$ and $b_{i} \geq 13$. Let $\beta$ denote the number of parts among $1,2, \ldots, 13$. If $\beta \geq 7$, then (3.6) violates $S$ or $S_{1}$. Hence, $1 \leq \beta \leq 6$. Let $\alpha$ denote the number of parts 13 so that $5 \leq \alpha \leq 10$ and $\alpha+\beta=11$. Then the number being partitioned is

$$
\left(12+x_{\alpha}\right)+\cdots+\left(12+x_{1}\right)+y_{1}+\cdots+y_{\beta}
$$

Since $\left(12+x_{\alpha}\right)-y_{\beta}<13$, we have $x_{\alpha}=y_{\beta}$. Now, $x_{1} \geq 2, x_{2} \geq 3, \ldots, x_{\alpha} \geq \alpha+1$. Thus, $y_{\beta} \geq \alpha+1, \ldots, y_{1} \geq \alpha+\beta=11$. Hence, (3.10) is greater than or equal to

$$
(12+\alpha+1)+\cdots+(12+2)+(\alpha+\beta)+\cdots+(\alpha+1)
$$


and equals

$$
\frac{13 \alpha+(\alpha+\beta)(\alpha+\beta+1)}{2}
$$

Let $\beta=1,2,3,4,5$. Then (3.10) is, respectively, $196,183,170,157$, and 144 , all of which are greater than $136=n^{c}$.

Now let $\beta=6$. Since we have to choose 6 parts from $1,2, \ldots, 13$ and 5 parts greater than 13 for a partition violating $S^{*}$ (and not violating any of $S, S_{1}, \ldots, S_{6}$ ), it is clear that the minimum part should be 6 . Let $S_{1}^{*}=\{6,7\}$ and $S_{2}^{*}=\{8,9,10,11,12,13\}$. Since $f_{6}+f_{7} \leq 1$, we can choose either 6 or 7 from $S_{1}^{*}$ and the other five must be from $S_{2}^{*}$. Also there are 5 parts greater than 13. In this case, the minimum value of $n$ will be

$$
6+8+9+10+11+12+14+15+16+17+18=136 .
$$

Thus for all $130 \leq n<136$, there are no partitions of $n$ violating only $S^{*}$. It is easy to see that when $n=136$,

$$
18+\cdots+14+12+\cdots+8+6
$$

is the only partition of 136 violating only $S^{*}$. Thus we find

$$
P_{A}^{\prime}(n)=\left\{\text { union of the partitions violating } S_{1}, \ldots, S_{6}\right\} \text { for } 1 \leq n<136
$$

while

$$
P_{A}^{\prime}(136)=\left\{\text { union of the partitions violating } S_{1}, \ldots, S_{6}\right\}+1 \text {. }
$$

We now establish a bijection of $Q^{1} \cup \cdots \cup Q^{6}$ onto $P_{A}^{\prime}(n)$ which is explained in Table 3.1. This also proves (3.3) and (3.4). Before writing the table, we observe that for a partition

$$
\pi+13 \times i+\alpha_{1}+\cdots+\alpha_{j}, \quad 1 \leq i \leq 6,
$$

belonging to $P_{B}^{\prime}, \pi$ is a partition of $\left(n-13 \times i-\alpha_{1}-\cdots-\alpha_{j}\right)$ into parts greater than 13 with $C$, where $1 \leq \alpha_{j}<\cdots<\alpha_{1} \leq 12$, and for a partition

$$
\pi+\beta_{1}+\cdots+\beta_{j}
$$

belonging to $P_{A}^{\prime}, \pi$ is a partition of $\left(n-\beta_{1}-\cdots-\beta_{j}\right)$ into parts greater than $\beta_{1}$ such that 13 is not a part, where $1 \leq \beta_{j}<\cdots<\beta_{1} \leq 12$.

REMARK 3.1. In Table 3.1, some partitions in $Q^{2}$ are not covered. They are

$$
\begin{aligned}
& \left\{\pi+13 \times 2+x_{1}+x_{2}+1: 2 \leq x_{2} \leq 11,3 \leq x_{1} \leq 12,\left(x_{1}, x_{2}\right) \neq(7,6)\right\} \\
& \quad \cup\left\{\pi+13 \times 2+12+x_{1}+x_{2}: 3 \leq x_{1} \leq 11,2 \leq x_{2} \leq 10,\left(x_{1}, x_{2}\right) \neq(7,6)\right\} .
\end{aligned}
$$

Here we split $13 \times 2$ into pairs $(\alpha, \beta)$ and $(\gamma, \delta)$ in the following order:

$$
(7,6)(8,5)(9,4)(10,3)(11,2)(12,1) \text {. }
$$


TABLE 3.1

\begin{tabular}{|c|c|}
\hline$P_{B_{12,11,7}}^{\prime}(n)$ & $P_{A_{12,11,7}}^{\prime}(n)$ \\
\hline$Q^{6}=\{\pi+13 \times 6\}$ & 1st stage of $S_{1}=\{\pi+12+\cdots+1\}$ \\
\hline$Q^{5}=\{\pi+13 \times 5\}$ & 1st stage of $S_{2}=\{\pi+11+\cdots+2\}$ \\
\hline$\cup\left\{\pi+13 \times 5+\left(13-x_{1}\right):\right.$ & 2nd stage of $S_{1}=\left\{\pi+12+\cdots+\left(x_{1}+1\right)\right.$ \\
\hline $\left.1 \leq\left(13-x_{1}\right) \leq 12\right\}$ & $\begin{array}{l}+\left(x_{1}-1\right)+\cdots+2+1: \\
\left.1 \leq x_{1} \leq 12\right\}\end{array}$ \\
\hline$Q^{4}=\{\pi+13 \times 4+x: x=0,1,2,12\}$ & $\begin{array}{c}\text { 1st stage of } S_{3}=\{\pi+10+\cdots+3+x: x \\
=0,1,2,12\}\end{array}$ \\
\hline$\cup\left\{\pi+13 \times 4+\left(13-x_{1}\right):\right.$ & 2nd stage of $S_{2}=\left\{\pi+11+\cdots+\left(x_{1}+1\right)\right.$ \\
\hline $\left.2<\left(13-x_{1}\right) \leq 11\right\}$ & $\left.+\left(x_{1}-1\right)+\cdots+2: 2 \leq x_{1}<11\right\}$ \\
\hline$\cup\left\{\pi+13 \times 4+\left(13-x_{1}\right)+\left(13-x_{2}\right):\right.$ & 3rd stage of $S_{1}=\left\{\pi+12+\cdots+\left(x_{1}+1\right)\right.$ \\
\hline $\left.1 \leq\left(13-x_{2}\right)<\left(13-x_{1}\right) \leq 12\right\}$ & $\begin{array}{l}\qquad \begin{array}{l}+\left(x_{1}-1\right)+\cdots+\left(x_{2}+1\right)+\left(x_{2}-1\right) \\
+\cdots+1: 1 \leq x_{2}<x_{1} \leq 12, \\
\left.\left(x_{i}, x_{j}\right) \neq(7,6)\right\}\end{array} \\
\text { Note 1. If }\left(x_{i}, x_{j}\right)=(7,6) \text {, then it will be covered } \\
\text { in the 3rd stage of } S_{2} \text {. }\end{array}$ \\
\hline$Q^{3}=\{\pi+13 \times 3+x: x=0,1,2,11,12\}$ & $\begin{array}{c}\text { 1st stage of } S_{4}=\{\pi+9+\cdots+4+x: x= \\
0,1,2,11,12\}\end{array}$ \\
\hline$\cup\left\{\pi+13 \times 3+\left(13-x_{1}\right):\right.$ & 2nd stage of $S_{3}=\left\{\pi+10+\cdots+\left(x_{1}+1\right)\right.$ \\
\hline $\left.3 \leq\left(13-x_{1}\right) \leq 10\right\}$ & $\left.+\left(x_{1}-1\right)+\cdots+3: 3 \leq x_{1} \leq 10\right\}$ \\
\hline$\cup\left\{\pi+13 \times 3+\left(13-x_{1}\right)+\left(13-x_{2}\right):\right.$ & 3rd stage of $S_{2}=\left\{\pi+11+\cdots+\left(x_{1}+1\right)\right.$ \\
\hline $\left.2 \leq\left(13-x_{2}\right)<\left(13-x_{1}\right) \leq 11\right\}$ & 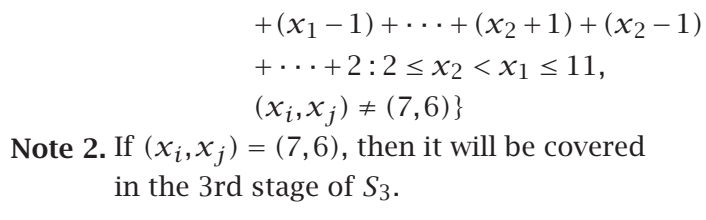 \\
\hline $\begin{array}{l}\cup\{\pi+13 \times 3+x+y:(x, y) \\
\quad=\text { all possible pairs of } 1,2,11,12 \\
\quad \text { except }(11,2)\}\end{array}$ & $\begin{aligned} 4 \text { th stage of } S_{4}=\{ & \pi+9+\cdots+4+x+y: \\
& (x, y)=\text { all possible pairs of } \\
& 1,2,11,12 \text { except }(11,2)\}\end{aligned}$ \\
\hline $\begin{array}{l}\cup\left\{\pi+13 \times 3+\left(13-x_{1}\right)+\cdots\right. \\
\quad+\left(13-x_{3}\right): 1 \leq\left(13-x_{3}\right) \\
\left.\quad<\left(13-x_{2}\right)<\left(13-x_{1}\right) \leq 12\right\}\end{array}$ & $\begin{array}{l}\text { 4th stage of } \begin{aligned} S_{1}=\{ & \pi+12+\cdots+\left(x_{1}+1\right) \\
& +\left(x_{1}-1\right)+\cdots+\left(x_{3}+1\right)+\left(x_{3}-1\right) \\
& +\cdots+1: 1 \leq x_{3}<x_{2}<x_{1} \leq 12, \\
& \left.\left(x_{i}, x_{j}\right) \neq(7,6)\right\}\end{aligned} \\
\text { Note 3. If }\left(x_{i}, x_{j}\right)=(7,6), \text { then it will be covered } \\
\text { in the 4th stage of } S_{2} .\end{array}$ \\
\hline $\begin{array}{c}Q^{2}=\{\pi+13 \times 2+x: x=0,1,2 \\
3,10,11,12\}\end{array}$ & $\begin{aligned} \text { 1st stage of } S_{5}=\{\pi+8+7+6+5+x: \\
\\
\qquad x=0,1,2,3,10,11,12\}\end{aligned}$ \\
\hline$\cup\left\{\pi+13 \times 2+\left(13-x_{1}\right):\right.$ & 2nd stage of $S_{4}=\left\{\pi+9+\cdots+\left(x_{1}+1\right)\right.$ \\
\hline $\left.4 \leq\left(13-x_{1}\right) \leq 9\right\}$ & $\left.+\left(x_{1}-1\right)+\cdots+4: 4 \leq x_{1} \leq 9\right\}$ \\
\hline$\cup\left\{\pi+13 \times 2+\left(13-x_{1}\right)+\left(13-x_{2}\right):\right.$ & 3rd stage of $S_{3}=\left\{\pi+10+\cdots+\left(x_{1}+1\right)\right.$ \\
\hline $\left.3 \leq\left(13-x_{2}\right)<\left(13-x_{1}\right) \leq 10\right\}$ & $\begin{array}{l}+\left(x_{1}-1\right)+\cdots+\left(x_{2}+1\right) \\
+\left(x_{2}-1\right)+\cdots+3 \\
: 3 \leq x_{2}<x_{1} \leq 10 \\
\left.\left(x_{i}, x_{j}\right) \neq(7,6)\right\}\end{array}$ \\
\hline
\end{tabular}


TABle 3.1. Continued.

\begin{tabular}{|c|c|}
\hline$P_{B_{12,11,7}^{\prime}}^{\prime}(n)$ & $P_{A_{12,11,7}}^{\prime}(n)$ \\
\hline & $\begin{array}{l}\text { Note 4. If }\left(x_{i}, x_{j}\right)=(7,6) \text {, then it will be covered in } \\
\text { the 3rd stage of } S_{4} \text {. }\end{array}$ \\
\hline $\begin{array}{l}\cup\{\pi+13 \times 2+x+y:(x, y) \\
\quad=\text { all possible pairs of } 1,2,3,10 \\
\quad 11,12 \text { except }(10,3)\}\end{array}$ & $\begin{aligned} 4 \text { th stage of } S_{5}=\{ & \pi+8+7+6+5+ \\
& x+y:(x, y)=\text { all possible } \\
& \text { pairs of } 1,2,3,10,11,12 \\
& \text { except }(10,3)\}\end{aligned}$ \\
\hline $\begin{array}{l}\cup\left\{\pi+13 \times 2+\left(13-x_{1}\right)+\cdots\right. \\
\quad+\left(13-x_{3}\right): 2 \leq\left(13-x_{3}\right) \\
\left.\quad<\left(13-x_{2}\right)<\left(13-x_{1}\right) \leq 11\right\}\end{array}$ & $\begin{aligned} 4 \text { th stage of } S_{2}=\{ & \pi+11+\cdots+\left(x_{1}+1\right) \\
& +\left(x_{1}-1\right)+\cdots+\left(x_{3}+1\right) \\
& +\left(x_{3}-1\right)+\cdots+2: 2 \leq x_{3} \\
& \left.<x_{2}<x_{1} \leq 11\right\} \\
& \left.\left(x_{i}, x_{j}\right) \neq(7,6)\right\}\end{aligned}$ \\
\hline & $\begin{array}{l}\text { Note 5. If }\left(x_{i}, x_{j}\right)=(7,6) \text {, then it will be } \\
\text { covered in the } 4 \text { th stage of } S_{3} .\end{array}$ \\
\hline $\begin{array}{l}\cup\left\{\pi+13 \times 2+\left(13-x_{1}\right)+\cdots\right. \\
\quad+\left(13-x_{4}\right): 1 \leq\left(13-x_{4}\right) \\
\left.\quad<\cdots<\left(13-x_{1}\right) \leq 12\right\}\end{array}$ & $\begin{aligned} \text { 5th stage of } S_{1}=\{ & \pi+12+\cdots+\left(x_{1}+1\right) \\
& +\left(x_{1}-1\right)+\cdots+\left(x_{4}+1\right) \\
& +\left(x_{4}-1\right)+\cdots+1: 1 \leq x_{4} \\
& \left.<\cdots<x_{1} \leq 12\right\} \\
& \left.\left(x_{i}, x_{j}\right) \neq(7,6)\right\}\end{aligned}$ \\
\hline & $\begin{array}{l}\text { Note 6. If }\left(x_{i}, x_{j}\right)=(7,6) \text {, then it will be } \\
\text { covered in the } 5 \text { th stage of } S_{2} \text {. }\end{array}$ \\
\hline$Q^{1}=\{\pi+13+x:$ & 1st stage of $S_{6}=\{\pi+7+6+x$ : \\
\hline$x=0,1,2,3,4,9,10,11,12\}$ & $x=0,1,2,3,4,9,10,11,12\}$ \\
\hline$\cup\left\{\pi+13+\left(13-x_{1}\right):\right.$ & 2nd stage of $S_{5}=\left\{\pi+8+\cdots+\left(x_{1}+1\right)\right.$ \\
\hline $\left.5 \leq\left(13-x_{1}\right) \leq 8\right\}$ & $\begin{aligned} & +\left(x_{1}-1\right)+\cdots+5 \\
& \left.: 5 \leq x_{1} \leq 8\right\}\end{aligned}$ \\
\hline $\begin{array}{r}\cup\left\{\pi+13+\left(13-x_{1}\right)+\left(13-x_{2}\right):\right. \\
\left.\quad 4 \leq\left(13-x_{2}\right)<\left(13-x_{1}\right) \leq 9\right\}\end{array}$ & $\begin{aligned} \text { 3rd stage of } S_{4}=\{ & \pi+9+\cdots+\left(x_{1}+1\right) \\
& +\left(x_{1}-1\right)+\cdots+\left(x_{2}+1\right) \\
& +\left(x_{2}-1\right)+\cdots+4: 4 \leq x_{2} \\
& \left.<x_{1} \leq 9\right\}\end{aligned}$ \\
\hline$\cup\{\pi+13+x+y:(x, y)$ & 4th stage of $S_{5}=\{\pi+7+6+x+y:(x, y)$ \\
\hline $\begin{array}{l}=\text { all possible pairs of } 1,2,3,4,9 \\
10,11,12 \text { except }(9,4)\}\end{array}$ & $\begin{array}{l}=\text { all possible pairs of } 1,2, \\
3,4,9,10,11,12 \text { except }(9,4)\}\end{array}$ \\
\hline$\cup\left\{\pi+13+\left(13-x_{1}\right)+\cdots\right.$ & 4th stage of $S_{3}=\left\{\pi+10+\cdots+\left(x_{1}+1\right)\right.$ \\
\hline $\begin{array}{l}+\left(13-x_{3}\right): 3 \leq\left(13-x_{3}\right) \\
\left.<\left(13-x_{2}\right)<\left(13-x_{1}\right) \leq 10\right\}\end{array}$ & $\begin{array}{l}+\left(x_{1}-1\right)+\cdots+\left(x_{3}+1\right) \\
+\left(x_{3}-1\right)+\cdots+3: 3 \leq x_{3} \\
\left.<x_{2}<x_{1} \leq 10\right\}\end{array}$ \\
\hline$\cup\left\{\pi+13+\left(13-x_{1}\right)+\cdots+\left(13-x_{4}\right):\right.$ & 5th stage of $S_{2}=\left\{\pi+11+\cdots+\left(x_{1}+1\right)\right.$ \\
\hline $\begin{array}{l}2 \leq\left(13-x_{4}\right)<\cdots<\left(13-x_{1}\right) \\
\leq 11\}\end{array}$ & $\begin{array}{l}+\left(x_{1}-1\right)+\cdots+\left(x_{4}+1\right) \\
+\left(x_{4}-1\right)+\cdots+2: 2 \leq x_{4} \\
\left.<\cdots<x_{1} \leq 11\right\}\end{array}$ \\
\hline $\begin{array}{l}\cup\left\{\pi+13+\left(13-x_{1}\right)+\cdots+\left(13-x_{5}\right):\right. \\
\quad 1 \leq\left(13-x_{5}\right)<\cdots<\left(13-x_{1}\right) \\
\quad \leq 12\}\end{array}$ & $\begin{aligned} 6 \text { th stage of } S_{1}=\{ & \pi+12+\cdots+\left(x_{1}+1\right) \\
& +\left(x_{1}-1\right)+\cdots+\left(x_{5}+1\right) \\
& +\left(x_{5}-1\right)+\cdots+1: 1 \leq x_{5} \\
& \left.<\cdots<x_{1} \leq 12\right\}\end{aligned}$ \\
\hline
\end{tabular}


We arrange $\pi+\alpha+\beta+\gamma+\delta+x_{1}+x_{2}+y(y=12$ or 1$)$ in the decreasing order and associate it to the rearranged partition $\pi^{*}$ which belongs to $P_{A}^{\prime}$.

A similar procedure is adopted for some partitions in $Q^{1}$ which are also not covered in Table 3.1. This completes the proof of (3.3) and (3.4).

\section{REFERENCES}

[1] G. E. Andrews, A generalization of the Göllnitz-Gordon partition theorems, Proc. Amer. Math. Soc. 18 (1967), 945-952.

[2] _ A generalization of the classical partition theorems, Trans. Amer. Math. Soc. 145 (1969), 205-221.

[3] _ Partition identities, Adv. Math. 9 (1972), 10-51.

[4] - On the General Rogers-Ramanujan Theorem, Memiors of the American Mathematical Society, no. 152, American Mathematical Society, Rhode Island, 1974.

[5] G. E. Andrews, C. Bessenrodt, and J. B. Olsson, Partition identities and labels for some modular characters, Trans. Amer. Math. Soc. 344 (1994), no. 2, 597-615.

[6] H. Göllnitz, Partitionen mit differenzenbedingungen, J. Reine Angew. Math. 225 (1967), 154-190 (German).

[7] B. Gordon, A combinatorial generalization of the Rogers-Ramanujan identities, Amer. J. Math. 83 (1961), 393-399.

[8] Padmavathamma and M. R. Salestina, On a conjecture of Andrews. II, Number Theory and Discrete Mathematics. Proceedings of the International Conference in Honour of Srinivasa Ramanujan (Chandigarh, 2000), Trends Math., Birkhäuser, Basel, 2002, pp. 135-147.

[9] Padmavathamma and T. G. Sudha, On a conjecture of Andrews, Int. J. Math. Math. Sci. 16 (1993), no. 4, 763-774.

[10] I. J. Schur, Zur additiven zahlentheorie, Sitzungsber. Preuss. Akad. Wiss. Phys.-Math. KI (1926), 488-495 (German).

Padmavathamma: Department of Studies in Mathematics, University of Mysore, Manasagangotri, Mysore 570 006, India

E-mail address: padma_vathamma@yahoo.com

M. R. Salestina: Department of Studies in Mathematics, University of Mysore, Manasagangotri, Mysore 570 006, India

E-mail address: sa1estina@rediffmai1.com 


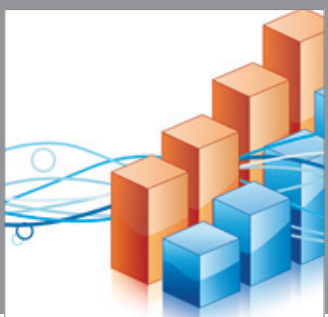

Advances in

Operations Research

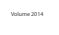

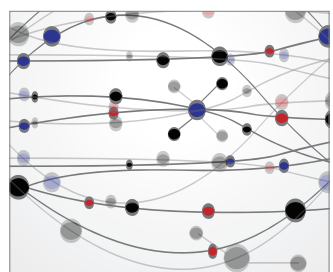

\section{The Scientific} World Journal
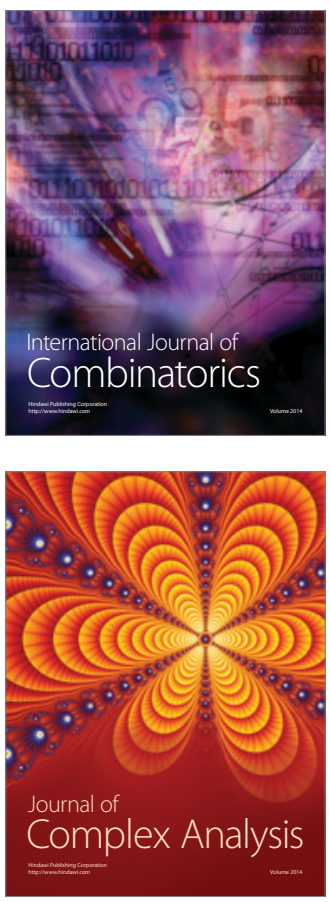

International Journal of

Mathematics and

Mathematical

Sciences
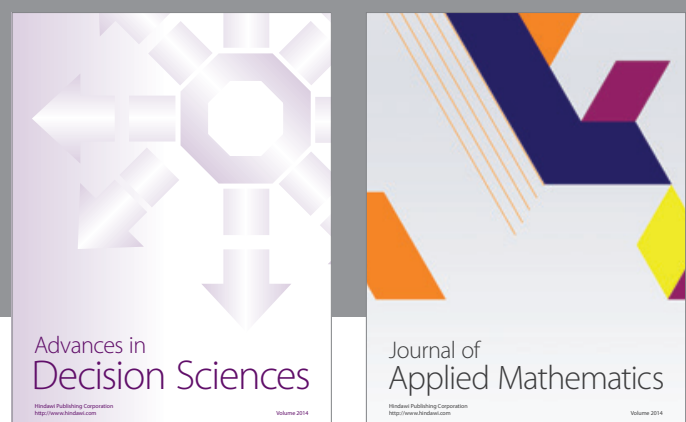

Journal of

Applied Mathematics
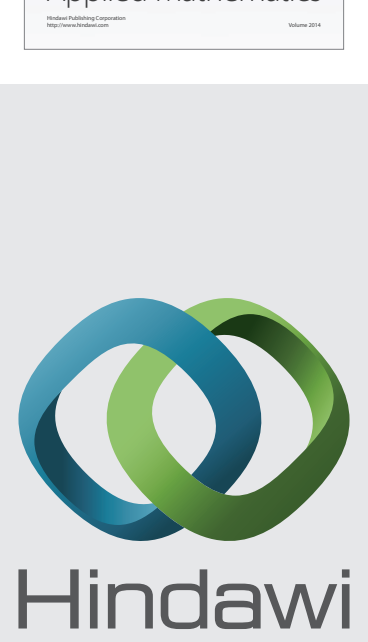

Submit your manuscripts at http://www.hindawi.com
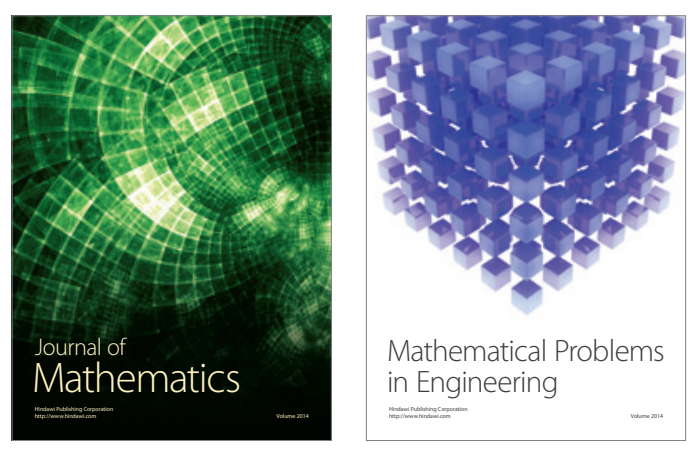

Mathematical Problems in Engineering
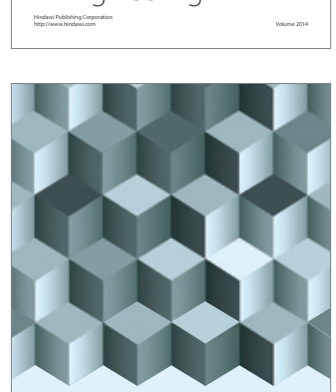

Journal of

Function Spaces
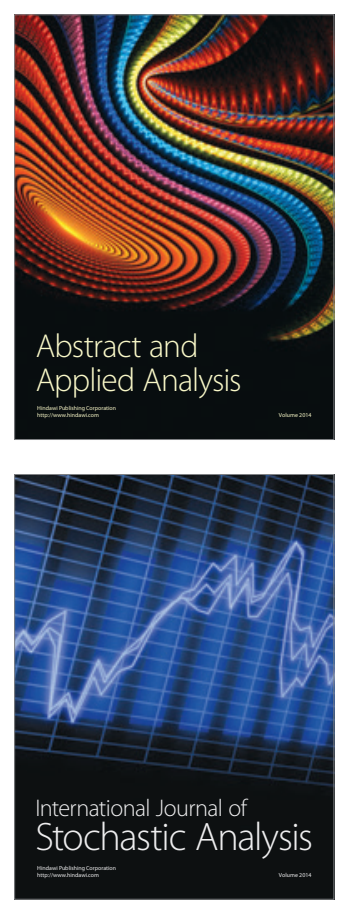

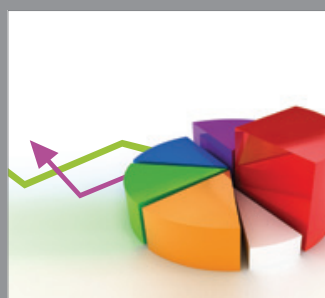

ournal of

Probability and Statistics

Promensencen
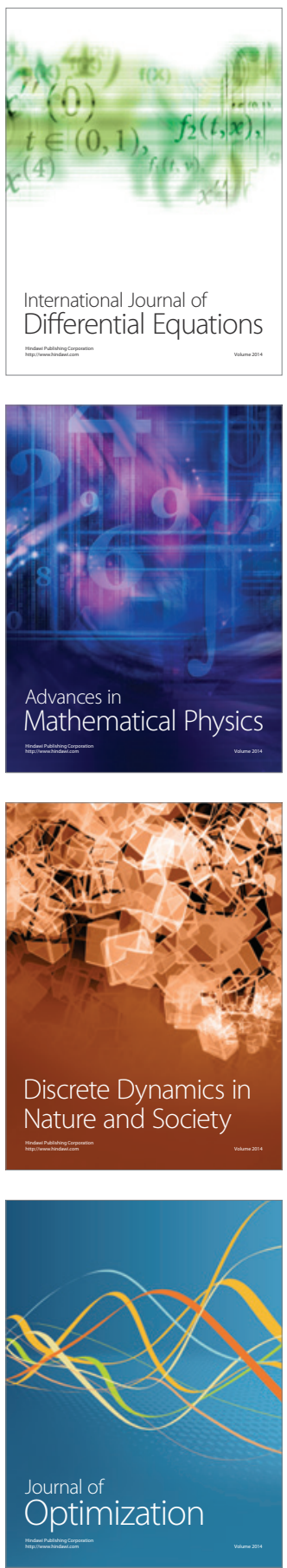